\title{
ESTUDO DA CINÉTICA E DO EQUILÍBRIO DE ADSORÇÃO DO CORANTE AZUL REATIVO 5G UTILIZANDO ESCAMA DE PEIXE COMO ADSORVENTE
}

\author{
C. V. NEVES*, A. P. NARDINO, K. C. KERBER, C. RIBEIRO, C. E. BORBA \\ Universidade Estadual do Oeste do Paraná, Departamento de Engenharia Química \\ "e-mail: camilaneves_@hotmail.com
}

\begin{abstract}
RESUMO
Os corantes estão presentes em altas concentrações no efluente têxtil e boa parte é descarregada em corpos hídricos devido a sua baixa biodegradabilidade e alta solubilidade. Desta forma, o efluente têxtil precisa ser tratado antes de despeja-lo no meio ambiente e o processo de adsorção pode ser um método eficiente, de fácil operação e de baixo custo, principalmente, se o adsorvente utilizado for subproduto industrial. Neste sentido, este trabalho tem como objetivo a obtenção da cinética e o equilíbrio de adsorção do corante reativo azul 5G (RB5G) utilizando escamas de peixe do gênero tilápia como adsorvente. Os experimentos de adsorção foram realizados em sistema fechado e batelada, em $\mathrm{pH} 2$ e temperatura de $25^{\circ} \mathrm{C}$. Os dados de equilíbrio de adsorção foram modelados a partir da isoterma de Langmuir e foi obtida uma capacidade máxima de adsorção de $272,39 \mathrm{mg} / \mathrm{g}$. Os dados da cinética de adsorção foram obtidos nas concentrações iniciais de RB5G de 25, 50, 100, 150 e $200 \mathrm{mg} \mathrm{L}^{-1}$, respectivamente e o tempo de equilíbrio alcançado foi de 72 horas. A cinética de adsorção foi descrita pelo modelo de adsorção-dessorção de Langmuir, o qual se ajustou bem aos dados experimentais. Desta forma, a remoção do corante RB5G pela escama de peixe se mostrou eficiente, a qual apresentou capacidade de adsorção elevada. Os modelos ajustados aos dados cinéticos e de equilíbrio de adsorção descreveram bem os dados experimentais, assim, as informações fornecidas pelos modelos podem ser utilizadas no projeto deste processo em escala piloto.
\end{abstract}

\section{INTRODUÇÃO}

O crescimento populacional das últimas décadas tem intensificado as atividades agrícolas, a industrialização e também as mudanças ambientais, fatores que são responsáveis por deteriorar continuamente os recursos aquáticos (ZHANG, 2013). Diversos tipos de poluentes orgânicos e inorgânicos são despejados em corpos aquáticos, contaminando-os, muitas vezes de forma irreversível. O setor têxtil é um dos grandes poluidores, devido ao grande volume de água utilizado no processo produtivo e pela utilização de altas concentrações de corantes na etapa de acabamento (AUONI et al., 2012).

O consumo total de corantes na indústria têxtil a nível mundial é mais de 10 mil toneladas/ano e cerca de 100 toneladas/ano de corantes são descarregadas em corpos hídricos (YAGUB, 2012). Os corantes são moléculas de baixa biodegradabilidade e alta solubilidade dificultando sua remoção das águas residuárias, além de serem tóxicos tanto ao homem quanto ao meio ambiente. Desta forma, corantes presentes em águas 
residuárias precisam ser removidos antes do descarte do efluente no meio ambiente.

Os tratamentos convencionais que têm sido aplicados a efluentes têxteis são eletrocoagulação (PALACIO et al., 2009), processos oxidativos avançados (MANENTI et al., 2010), biológicos (MANIMEKALAI \& SWAMINATHAN, 2000), separação por membranas (SU et al., 2009), entre outros. Estes processos são eficazes, como mostram as pesquisas, no entanto requerem uma combinação de dois ou mais métodos para remoção adequada dos corantes (VERMA et $a l .$, 2012). Em contrapartida, a adsorção pode associar eficiência, facilidade de operação e baixo custo, principalmente, quando se utiliza subprodutos industriais como adsorvente (SALLEH et al., 2011).

$\mathrm{Na}$ adsorção, o estudo do equilíbrio e da cinética são fundamentais, pois fornecem informações sobre a capacidade máxima de adsorção do adsorvente, bem como sobre mecanismos de adsorção, o tempo de equilíbrio, interações entre adsorbatoadsorvente, entre outros.

Este trabalho tem como objetivo avaliar a cinética e o equilíbrio de adsorção do corante RB5G pelas escamas de peixe do gênero tilápia, bem como descrever estes dados por meio de modelagem matemática.

\section{PROCEDIMENTO EXPERIMENTAL}

\subsection{Preparo de soluções e adsorvente}

As soluções foram preparadas a partir da dissolução do corante reativo azul $5 \mathrm{G}$ em água destilada. $\mathrm{O} \mathrm{pH}$ inicial das soluções foi ajustado em 2 utilizando $\mathrm{HCl} \quad 0,1 \mathrm{M}$. O adsorvente utilizado para os experimentos de adsorção foi a escama de peixe inteira do gênero tilápia (Oreochromis niloticus), que foram previamente lavadas com água destilada e secas em estufa a uma temperatura de $60^{\circ} \mathrm{C}$ e armazenadas em freezer $\left(-18^{\circ} \mathrm{C}\right)$ até sua utilização nos experimentos.

\subsection{Experimentos de Adsorção}

Os experimentos de adsorção foram realizados em sistema fechado e batelada, contendo solução de corante RB5G em pH 2 e adsorvente, sob agitação e temperatura $\left(25^{\circ} \mathrm{C}\right)$ controladas. Ao final de cada experimento, foram retiradas alíquotas da fase líquida e a concentração de corante RB5G foi determinada por espectrômetro Uv-vis (UV 1800 Shimadzu) no comprimento de onda de $620 \mathrm{~nm}$.

\subsubsection{Cinética de Adsorção}

As cinéticas de adsorção do corante RB5G pela escama de peixe foram realizadas em um reator com controle de temperatura e de agitação mecânica. Um volume de solução do corante RB5G (1500 mL) e uma determinada massa (base seca) do adsorvente foram colocadas no reator e mantidos sob agitação e temperatura controladas. Foram realizados experimentos nas concentrações iniciais de corante de 200, 150, 100, 50 e 25

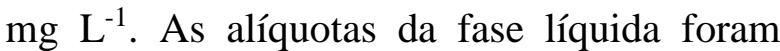
retiradas em tempos pré-determinados, até o tempo de equilíbrio (72 horas). O cálculo da quantidade adsorvida de corante pela escama de peixe foi realizado com base no balanço de massa, representado pela Equação 01.

$q(t)=\frac{V\left(C_{0}-C(t)\right)}{m}$

\subsubsection{Equilíbrio de Adsorção}

$O$ teste de equilíbrio foi realizado utilizando uma incubadora com agitação orbital e controle de temperatura (shaker). Em frascos erlenmeyers foram adicionados a solução de corante RB5G e adsorvente e, então, mantidos sob agitação constante (150 $\mathrm{rpm})$ e temperatura controlada $\left(25^{\circ} \mathrm{C}\right)$ até o tempo de equilíbrio ( 72 horas). O tempo de equilíbrio foi baseado nos testes cinéticos. Os experimentos foram realizados em triplicata. 
A concentração do corante RB5G no adsorvente no equilíbrio foi calculada pela Equação 2:

$$
q_{e q}=\frac{V\left(C_{0}-C_{e q}\right)}{m}
$$

\section{MODELAGEM MATEMÁTICA}

\subsection{Isotermas de Adsorção}

Para descrever o equilíbrio de adsorção foi utilizado o modelo de isoterma de Langmuir, o qual se baseia nas hipóteses de que a superfície do sólido possui um número definido de sítios para adsorção, cada sítio é capaz de adsorver uma única molécula, a adsorção ocorre em uma única camada no adsorvente e não existe interação entre as moléculas adsorvidas.

A expressão matemática para isoterma de Langmuir é dada pela Equação 3.

$$
q_{e q}=\frac{q_{\max } b C_{e q}}{1+b C_{e q}}
$$

\subsection{Cinéticas de Adsorção}

Os modelos cinéticos são baseados em balanços de massa na fase líquida e na fase sólida, o qual descreve os mecanismos de transferência de massa no adsorvente (CHAHBANI; TONDEUR, 2000). Estas equações associam a variação da concentração em relação ao tempo de uma dada espécie no adsorvente, as quais representam a etapa controladora do processo de transferência de massa.

O modelo cinético apresentado pela Equação 4 é o de adsorção-dessorção de Langmuir, o qual considera como etapa limitante da transferência de massa a etapa de adsorção do corante RB5G na superfície do adsorvente.

$$
\frac{d q}{d t}=K_{a d s} C\left(q_{\max }-q\right)-K_{d e s} q
$$

No equilíbrio ( $d q / d t=0$ ), a Equação 4 se transforma na isoterma de Langmuir, sendo que o parâmetro $b$ é a razão entre a constante cinética de adsorção e constante cinética de dessorção. Assim, a Equação 4 pode ser escrita como:

$\frac{d q}{d t}=k_{a d s} C\left(q_{\max }-q\right)-\frac{k_{a d s}}{b} q$

As condições iniciais são representadas pelas Equações 6 e 7.

$$
\begin{aligned}
& C(0)=C_{0} \\
& q(0)=0
\end{aligned}
$$

\subsection{Procedimento para Estimativa dos Parâmetros}

Os parâmetros da isoterma de Langmuir ( $q_{\max } \mathrm{e} b$ ) foram estimados a partir do ajuste do modelo aos dados experimentais de equilíbrio, com base na minimização da função objetivo representada pela Equação 8.

$$
O F=\sum_{j=1}^{N}\left(\left(\frac{q_{j}^{\exp }-q_{j}^{\bmod }}{q_{\exp }^{\max }}\right) * \delta_{j}\right)^{2}
$$

$\mathrm{O}$ parâmetro do modelo cinético $\left(\mathrm{k}_{\mathrm{ads}}\right)$ foi estimado a partir do ajuste do modelo aos dados experimentais da cinética de adsorção, com base na minimização da função objetivo representada pela Equação 9.

$O F=\sum_{j=1}^{N}\left(\frac{C_{j}^{\exp }-C_{j}^{\bmod }}{C_{j}^{\exp }}\right)^{2}$

O método de otimização desenvolvido por Nelder e Mead (1965) foi utilizado para busca do mínimo da função objetivo. $\mathrm{O}$ método de Rosenbrock foi utilizado para resolver as equações diferenciais do modelo 
cinético. Os programas foram desenvolvidos com o auxílio do software Maple ${ }^{\circledR}$.

\section{RESULTADOS E DISCUSSÕES}

\subsection{Equilíbrio de Adsorção}

Os dados experimentais de equilíbrio foram obtidos em sistema fechado e batelada. Na Figura 01 são apresentados os dados de equilíbrio, da adsorção do corante RB5G pela escama de peixe, experimentais e simulados pela isoterma de Langmuir. Baseado no coeficiente de determinação, apresentado na Tabela 01, a isoterma de Langmuir descreveu bem os dados experimentais.

Figura 1 - Isoterma de adsorção do corante RB5G pela escama de peixe.

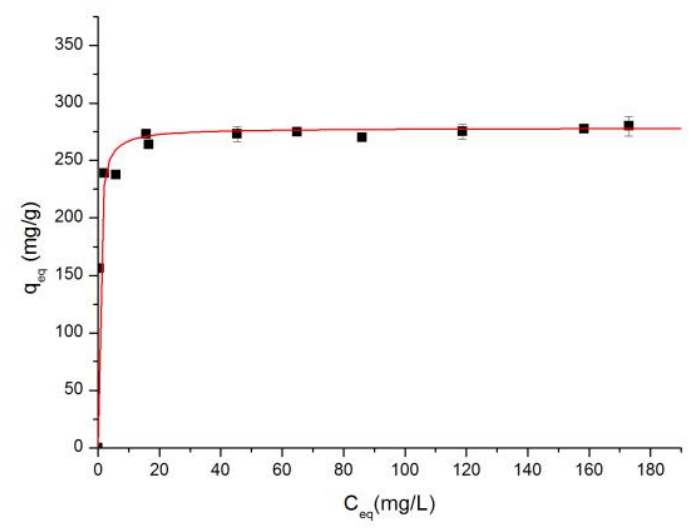

Os valores dos parâmetros ajustados da isoterma estão apresentados na Tabela 01. A capacidade máxima de adsorção do corante RB5G pela escama de peixe foi de $272,39 \mathrm{mg}$ $\mathrm{g}^{-1}$. RIBEIRO et al., (2015) encontrou um valor próximo de $\mathrm{q}_{\max }=230,00 \mathrm{mg} \mathrm{g}^{-1}$, para o mesmo corante (RB5G) e como adsorvente, foi utilizado escama de peixe em pó. Estes, são valores considerados altos quando comparados a outros adsorventes, visto que FIORENTIN et al., (2010) estudou a remoção do corante RB5G utilizando bagaço de laranja e a capacidade máxima obtida foi de $\mathrm{q}_{\max }=34,89 \mathrm{mg} \mathrm{g}^{-1}$. Além disto, verificou-se que o processo de adsorção é favorável, pois a inclinação da curva mostra que, mesmo em baixas concentrações a remoção de corante RB5G é alta. O parâmetro b indica a afinidade entre o adsorbato-adsorvente, ou seja, quanto maior este valor, mais afinidade há entre os íons e os sítios do adsorvente.

Tabela 1 - Valores dos parâmetros da isoterma de Langmuir.

\begin{tabular}{cc}
\hline \multicolumn{2}{c}{ Isoterma de Langmuir } \\
\hline $\mathrm{q}_{\max }\left(\mathrm{mg} \mathrm{g}^{-1}\right)$ & 272,39 \\
$\mathrm{~b}\left(\mathrm{~L} / \mathrm{mg}^{2}\right)$ & 2,61 \\
$\mathrm{R}^{\mathbf{2}}$ & 0,9953
\end{tabular}

\subsection{Cinética de Adsorção}

Na Figura 2 são apresentados os dados cinéticos experimentais e simulados, para as diferentes concentrações iniciais do corante RB5G na fase líquida. O tempo de equilíbrio, para todas as concentrações, ficou entre 4000 e $6000 \mathrm{~min}$. No entanto, verifica-se uma maior inclinação da parte inicial das curvas cinéticas a medida que a concentração inicial de corante na fase líquida é aumentada. Isto se deve a transferência de massa estar relacionada ao gradiente de concentração, ou seja, quanto maior a concentração de corante RB5G, maior a transferência de massa.

O modelo cinético de adsorçãodessorção de Langmuir foi escolhido para descrever os dados pelo fato do tamanho da molécula de corante RB5G (comprimento longitudinal $=22,02 \AA$, diâmetro médio = $10,68 \AA$ ) ser maior que o tamanho dos poros presentes no adsorvente $(20,6 \AA)$, conforme identificou RIBEIRO et al., (2015). Desta forma, acredita-se que a alta capacidade de adsorção da escama de peixe pelo corante RB5G esteja relacionado a adsorção na superfície do adsorvente.

$\mathrm{Na}$ Tabela 02 são apresentados os valores do parâmetro do modelo ajustado (constante cinética de adsorção), bem como os respectivos valores de coeficiente de determinação. Estes, indicam que o modelo 
de adsorção na superfície descreve bem os dados experimentais, sendo os valores de $\mathrm{R}^{2}$ acima de 0,98 . Os valores encontrados para a constante cinética de adsorção ( $\left.\mathrm{k}_{\mathrm{ads}}\right)$ se mantiveram na mesma ordem de grandeza.

Figura 2 - Cinéticas de adsorção do corante RB5G pela escama de peixe em diferentes concentrações. A: $\mathrm{C}_{0}=25$ ppm, B: $\mathrm{C}_{0}=50$ ppm, C: $\mathrm{C}_{0}=100 \mathrm{ppm}, \mathrm{D}: \mathrm{C}_{0}=150 \mathrm{ppm}, \mathrm{E}: \mathrm{C}_{0}=200 \mathrm{ppm}$.
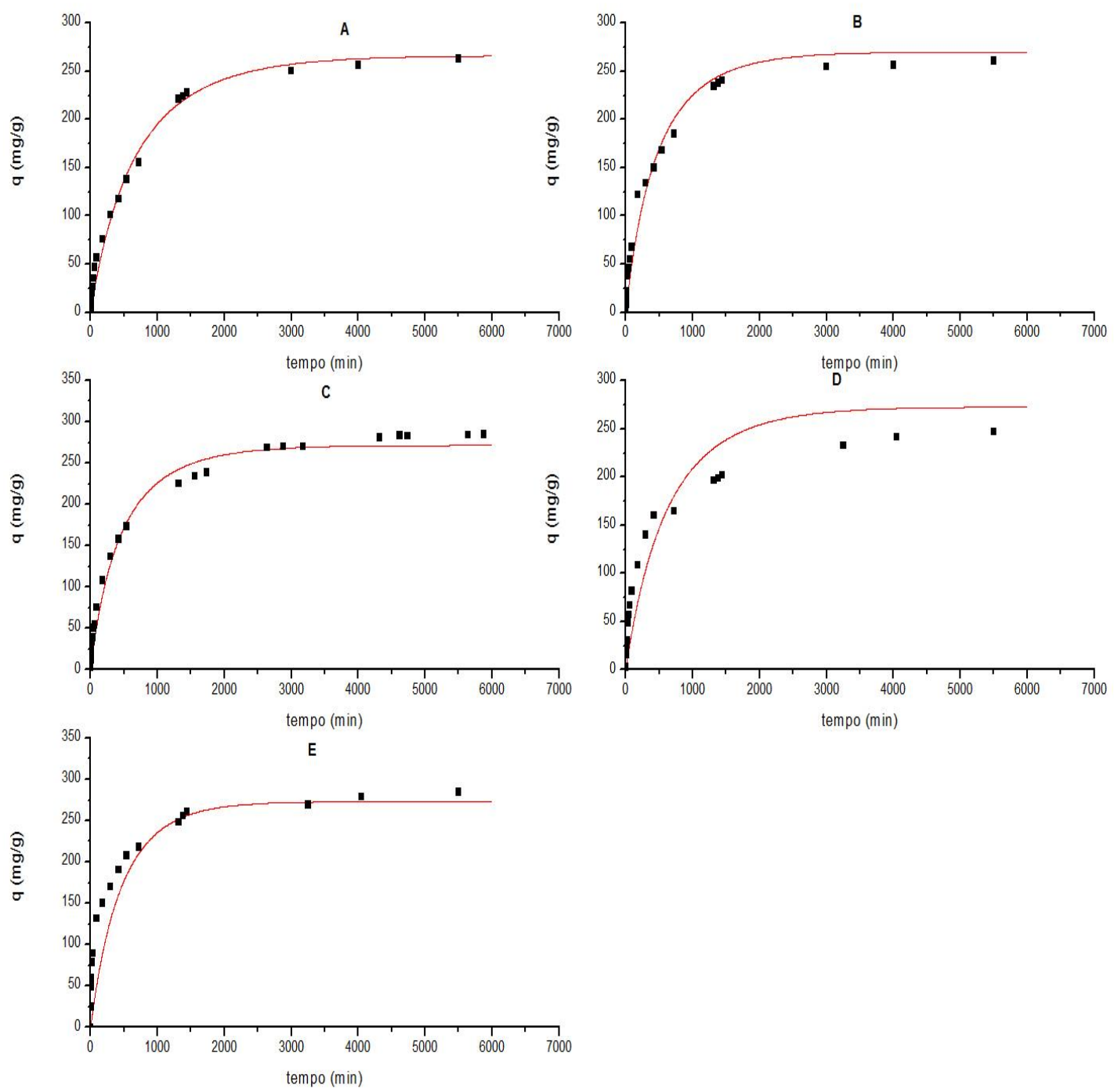
Tabela 2 - Valores dos parâmetros do modelo cinético de adsorção na superfície.

\begin{tabular}{ccc}
\hline $\begin{array}{c}\text { Concentração } \\
\text { inicial RB5G }(\mathrm{mg} \\
\left.\mathrm{L}^{-1}\right)\end{array}$ & $\begin{array}{c}\mathrm{k}_{\mathrm{ads}} \\
(\end{array}$ & $\mathrm{R}^{2}$ \\
\hline 25 & 0,000065 & 0,99 \\
50 & 0,000048 & 0,99 \\
100 & 0,000026 & 0,99 \\
150 & 0,000012 & 0,98 \\
200 & 0,000012 & 0,98
\end{tabular}

Segundo Coates (1969), Walker e Weatherley (2001), moléculas orgânicas como os corantes, podem se aglomerar em solução aquosa, especialmente em níveis de concentração elevados. Estes aglomerados são função da concentração de corante e podem formar dímeros, trímeros e até micelas com massa molecular elevada. Desta forma, os corantes podem adsorver tanto na forma aglomerada quanto em uma única molécula nos sítios disponíveis no adsorvente.

A Figura 3 apresenta a relação entre a constante cinética de adsorção $\left(\mathrm{k}_{\mathrm{ads}}\right)$ e a concentração inicial de RB5G. É possível notar que na faixa de concentração de 25 a $150 \mathrm{mg} \mathrm{L}^{-1}$ houve um decréscimo linear da constante cinética de adsorção ( $\mathrm{k}_{\mathrm{ads}}$ ). Isto pode ser devido a mudança do estado de aglomeração das moléculas de corante.

Entre as concentrações de corante de 150 a $200 \mathrm{mg} \mathrm{L}^{-1}$ a constante cinética de adsorção não se alterou. Possivelmente, a aglomeração das moléculas de corante nesta faixa de concentração foi a mesma.
Figura 3 - Dados de constante cinética de adsorção $\left(\mathrm{k}_{\mathrm{ads}}\right)$ em relação a concentração inicial de corante RB5G.

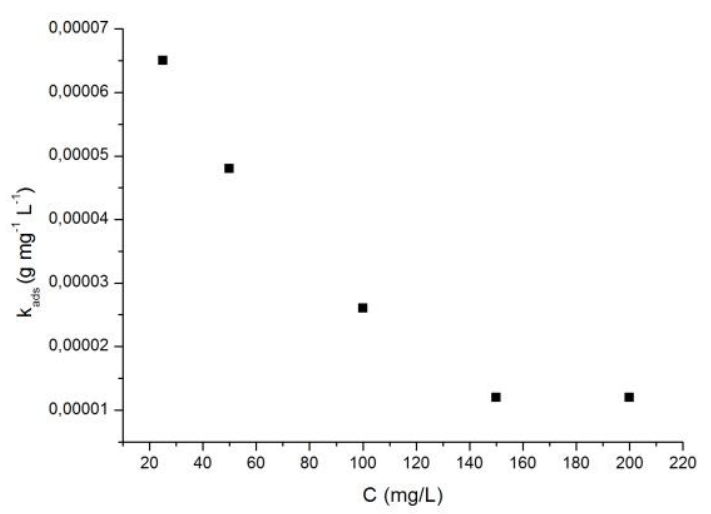

A modelagem matemática tanto dos dados cinéticos quanto dos dados de equilíbrio se ajustou bem aos dados experimentais, assim, as informações fornecidas pelos modelos podem ser utilizadas no projeto deste processo em escala piloto.

\section{CONCLUSÕES}

Neste trabalho foi avaliado a cinética e o equilíbrio de adsorção do corante RB5G pela escama de peixe. Os dados de equilíbrio indicaram que o adsorvente apresenta altas capacidades de remoção e o processo de adsorção é favorável, pois mesmo em baixas concentrações a adsorção é elevada. Os dados cinéticos indicaram um tempo de equilíbrio entre 4000 a $6000 \mathrm{~min}$.

$\mathrm{O}$ modelo de isoterma de Langmuir se ajustou bem aos dados de equilíbrio com $\mathrm{R}^{2}=$ 0,9953 . Além disso, o valor de $\mathrm{q}_{\max }$ foi de 272,39 $\mathrm{mg} \mathrm{g}^{-1}$, evidenciando o grande potencial de adsorção da escama de peixe.

O modelo cinético, baseado na cinética de adsorção-dessorção de Langmuir, foi utilizado para representar os dados experimentais. Os valores da constante cinética de adsorção $\left(\mathrm{k}_{\mathrm{ads}}\right)$ se mantiveram na 
mesma ordem de grandeza e foram diminuindo conforme o aumento da concentração de corante RB5G. Este modelo representou bem os dados experimentais com valores de $\mathrm{R}^{2}$ acima de 0,98 .

Assim, as informações obtidas pela modelagem matemática dos dados cinéticos e de equilíbrio servirão de base para representar um sistema em escala piloto.

\section{NOMENCLATURA}

$\mathrm{b}=$ constante de equilíbrio de adsorção de Langmuir em $\left(\mathrm{L} \mathrm{mg}^{-1}\right)$

$\mathrm{C}_{0}=$ concentração inicial de corante RB5G na fase líquida $\left(\mathrm{mg} \mathrm{L}^{-1}\right)$

$\mathrm{C}(\mathrm{t})=$ concentração de corante RB5G na fase líquida em determinado instante de tempo $\left(\mathrm{mg} \mathrm{L}^{-1}\right)$

$\mathrm{C}_{\text {eq }}=$ concentração de equilíbrio na fase líquida $\left(\mathrm{mg} \mathrm{L}^{-1}\right)$

$C_{j}^{\exp }=$ concentração de corante RB5G experimental na fase líquida $\left(\mathrm{mg} \mathrm{L}^{-1}\right)$ $C_{j}^{\bmod }$ $C_{j}=$ a concentração de corante RB5G obtido pelo modelo cinético $\left(\mathrm{mg} \mathrm{L}^{-1}\right)$

$k_{a d s}=$ constante cinética de adsorção $\left(\mathrm{g} \mathrm{mg}^{-1}\right.$ $\left.\mathrm{L}^{-1}\right)$

$k_{\text {des }}$ é a constante cinética de dessorção $\left(\mathrm{g} \mathrm{L}^{-2}\right)$

$\mathrm{m}=$ massa de adsorvente $(\mathrm{g})$

$\mathrm{N}=$ número de dados

$\mathrm{q}_{\mathrm{eq}}=$ quantidade de corante adsorvida na fase sólida $\left(\mathrm{mg} \mathrm{g}^{-1}\right)$

$\mathrm{q}_{\max }=$ quantidade máxima de corante adsorvida na fase sólida $\left(\mathrm{mg} \mathrm{g}^{-1}\right)$

$\mathrm{q}(\mathrm{t})$ = quantidade de corante adsorvida em determinado instante de tempo na fase sólida $\left(\mathrm{mg} \mathrm{g}^{-1}\right)$

$q_{j}^{\exp }=$ concentração experimental de corante RB5G no adsorvente no equilíbrio ( $\mathrm{mg} \mathrm{g}^{-1}$ )

$q_{j}^{\text {mod }}=$ concentração de corante RB5G no adsorvente no equilíbrio pela isoterma de Langmuir $\left(\mathrm{mg} \mathrm{g}^{-1}\right)$ $q_{\exp }^{\max }=$ máxima concentração de corante RB5G no adsorvente obtida experimentalmente $\left(\mathrm{mg} \mathrm{g}^{-1}\right)$

$\mathrm{V}=$ volume de solução (L)

$\delta_{j}=$ desvio padrão dos dados experimentais

\section{REFERÊNCIAS}

CHAHBANI, M.H., TONDEUR, D.; Mass transfer kinetics inpressure swing adsorption. Separation and Purification Technology, v. 20, p. 185-196, 2000.

COATES, E.; Aggregation of Dyes in Aqueous Solution. Journal of the Society of Dyers and Colourists, v. 157, p. 355-368, 1969.

FIORENTIN, L. D.; TRIGUEROS, D. E. G., MODENES, A. N.; ESPINOZA-QUIÑONES, F. R.; PEREIRA, N. C.; BARROSA, S. T. D; SANTOSA, O. A. A; Biosorption of reactive blue $5 \mathrm{G}$ dye onto drying orange bagasse in batch system: kinetic and equilibrium modeling. Chemical Engineering Journal, v. 163 , p. 68-77, 2010.

MANENTI D. R.; GOMES, L. F. S.; BORBA, F. H.; MÓDENES, A. N.; ESPINOZA-QUIÑNONES, F. R.; PALÁCIO, S. M.; Optimizing the Photo-Fenton Process using Artificial Irradiation on the Degradation of Synthetic Textile Effluent. Engevista, v. 12, p. 22-32, 2010.

MANIMEKALAI R.; SWAMINATHAN, T.; Removal of Hazardous Compounds by Lignin Peroxidase from Phanerochaete chrysosporium. Bioprocess and Biosystems Engineering, v. 22, p. 29-33, 2000. 
NELDER J. A.; MEAD R.; A simplex method for function minimization. The Computer Journal. V. 7, p. 308-315, 1965.

PALÁCIO S. M.; ESPINOZA-QUIÑNONES, F. R.; MÓDENES, A. N.; OLIVEIRA, C. C.; BORBA, F. H.; SILVA JR, F. G.; Toxicity Assessment from Electro-coagulation Treated-textile Dye Wastewaters by Bioassays. Journal of Hazardous Materials, v. 172, p. 330-337, 2009.

RIBEIRO, C.; SCHEUFELE, F. B.; ESPINOZA-QUIÑONES, F. R.; MÓDENES, A. N.; SILVA, M. G. C.; VIEIRA, M. G. A.; BORBA, C. E.; Characterisation of Oreochromis niloticus fish scales and assessment of their potential on the adsorption of reactive blue $5 \mathrm{G}$ dye. Colloids and Surfaces A: Physicochemical and Engineering Aspects, In Progress, 2015.

SALLEH, M. A. M.; MAHMOUD, D. K.; KARIM, W. A. W. A.; IDRIS, A.; Cationic and anionic dye adsorption by agricultural solid wastes: a comprehensive review. Desalination, v. 280, p.1-13, 2011.

SU, S. N.; NIE, H. L.; ZHU, L. M.; CHEN, T. $\mathrm{X}$.; Optimization of adsorption conditions of papain on dye affinity membrane using response surface methodology. Bioresources Technology, v. 100, p. 2336-2340, 2009.

VERMA, A. K.; DASH, R. R.; BHUNIA, P.; A review on chemical coagulation/ flocculation technologies for removal of colour from textile wastewaters. Journal of Environmental Management, v.93, p.154168, 2012.
YAGUB, M.T.; SEM, T. K.; AFROZE S.; ANG, H. M.; Dye and its removal from aqueous solution by adsorption: A review. Advances in Colloid and Interface Science, v. 209, p. 172-184, 2014.

Walker, G.M., Weatherley, L.R., 2001. Adsorption of dyes from aqueous solution the effect of adsorbent pore size distribution and dye aggregation. Chemical Engineering Journal. v. 83, p. 201-206, 2001.

ZHANG, L.H.; SUN, Q.; LIU, D.H.; LU, A.H.; Magnetic hollow carbon nanospheres for removal of chromium ions, Journal of Materials Chemistry, v. 1, p. 9477-9483, 2013.

\section{AGRADECIMENTOS}

A Coordenação de Aperfeiçoamento de Pessoal de Nível Superior (CAPES), pelo apoio financeiro. 